\title{
A Role for the Clathrin Assembly Domain of AP180 in Synaptic Vesicle Endocytosis
}

\author{
Jennifer R. Morgan, ${ }^{1,3}$ Xiaojun Zhao, ${ }^{2}$ Mary Womack, ${ }^{1,3}$ Kondury Prasad, ${ }^{2,3}$ George J. Augustine,,$^{1,3}$ and \\ Eileen M. Lafer ${ }^{2,3}$ \\ ${ }^{1}$ Department of Neurobiology, Duke University Medical Center, Durham, North Carolina 27710, 2Department of Molecular \\ Medicine, Institute of Biotechnology, University of Texas Health Science Center at San Antonio, San Antonio, Texas \\ 78245, and ${ }^{3}$ Marine Biological Laboratory, Woods Hole, Massachusetts 02543
}

We have used the squid giant synapse to determine whether clathrin assembly by AP180 is important for synaptic vesicle endocytosis. The squid homolog of AP180 encodes a 751 amino acid protein with $40 \%$ sequence identity to mouse AP180. Alignment of squid AP180 with other AP180 homologs shows that amino acid identity was highest in the $\mathrm{N}$-terminal inositide-binding domain of the protein and weakest in the C-terminal clathrin assembly domain. Recombinant squid AP180 was able to assemble clathrin in vitro, suggesting a conserved three-dimensional structure that mediates clathrin assembly despite the divergent primary sequence of the C-terminal domain. Microinjection of the C-terminal domains of either mouse or squid AP180 into the giant presynaptic terminal of squid enhanced synaptic transmission. Conversely, a peptide from the C-terminal domain of squid AP180 that inhibited clathrin assembly in vitro completely blocked synaptic transmission when it was injected into the giant presynaptic terminal.
This inhibitory effect occurred over a time scale of minutes when the synapse was stimulated at low $(0.03 \mathrm{~Hz})$, physiological rates. Electron microscopic analysis revealed several structural changes consistent with the inhibition of synaptic vesicle endocytosis; peptide-injected terminals had far fewer synaptic vesicles, were depleted of coated vesicles, and had a larger plasma membrane perimeter than terminals injected with control solutions. In addition, the remaining synaptic vesicles were significantly larger in diameter. We conclude that the clathrin assembly domain of AP180 is important for synaptic vesicle recycling at physiological rates of activity and that assembly of clathrin by AP180 is necessary for maintaining a pool of releasable synaptic vesicles.

Key words: membrane retrieval; synaptic vesicle; coated vesicle; clathrin-mediated endocytosis; squid giant synapse; AP180 homologs
Synaptic transmission relies on exocytosis and endocytosis reactions that involve synaptic vesicles and occur locally within presynaptic terminals. Although much progress has been made in identifying the proteins underlying synaptic vesicle exocytosis, molecular analysis of endocytosis is less developed (Rothman, 1994; Scheller, 1995; Sudhof, 1995; Augustine et al., 1996; De Camilli and Takei, 1996). Part of the problem is that there is still debate about the vesicle trafficking pathway used during endocytosis. Heuser and Reese (1973) proposed that synaptic vesicle endocytosis is mediated by coated vesicles. In contrast, it has been argued that endocytosis proceeds via a mechanism that does not use coated vesicles (Ceccarelli et al., 1973, 1979; Palfrey and Artalejo, 1998).

Central to this debate is the role of a key component of coated

\footnotetext{
Received July 6, 1999; revised Aug. 8, 1999; accepted Sept. 14, 1999.

This work was supported by National Institutes of Health Grant NS29051, a grant from the Muscular Dystrophy Association, and a Howard Hughes Medical Institute faculty development award (to E.M.L.); and by National Institutes of Health Grant NS21624 and a Human Frontier Science Program grant (to G.J.A.). We thank De Dieu for superb technical assistance and Larry Hawkey for performing the election microscopy. We thank Dr. James Battey (National Institutes of Health, Bethesda, MD) for providing the squid cDNA libraries. The reported sequences have been deposited in GenBank and assigned accession numbers AF182339 for squid AP180 and AF182340 for Xenopus AP180-1.

Correspondence should be addressed to Dr. Eileen M. Lafer, Department of Molecular Medicine, Institute of Biotechnology, University of Texas Health Science Center at San Antonio, 15355 Lambda Drive, San Antonio, TX 78245. E-mail: Lafer@uthscsa.edu.

Copyright (C) 1999 Society for Neuroscience 0270-6474/99/1910201-12\$05.00/0
}

vesicles, the coat protein clathrin (Pearse, 1976; Keen, 1987; Maycox et al., 1992; Takei et al., 1996; Cremona and De Camilli, 1997). We have evaluated the mechanism of synaptic vesicle endocytosis by perturbing clathrin function at the squid giant synapse. Because the presynaptic terminal is so large (Young, 1939), it is possible to microinject specific macromolecules while monitoring the effect of the macromolecules on presynaptic function and structure (Burns and Augustine, 1999). To examine the role of clathrin-mediated processes in synaptic vesicle endocytosis, we have focused on the synapse-specific protein AP180 (previously called pp155, NP185, F1-20, and AP-3; Ahle and Ungewickell, 1986; Zhou et al., 1992, 1993; McMahon, 1999). In vitro, this protein binds to clathrin, assembles clathrin triskelia into three-dimensional cages that resemble the coats of coated vesicles (Lindner and Ungewickell, 1992), and can form such coats over uncoated vesicles (Prasad and Lippoldt, 1988). In addition to its interactions with clathrin, AP180 is a major cellular ligand for inositides (Norris et al., 1995; Ye et al., 1995; Hao et al., 1997). Recent experiments show that deletion of the Drosophila AP180 gene impairs synaptic transmission and depletes synaptic vesicles, consistent with a block of endocytosis (Zhang et al., 1998). However, the sequence of Drosophila AP180 is quite divergent from mammalian AP180, and it has not been characterized biochemically. Thus, it remains to be determined whether the mutant phenotype is caused by the loss of possible inositide binding, clathrin assembly, or some other biochemical activity of 


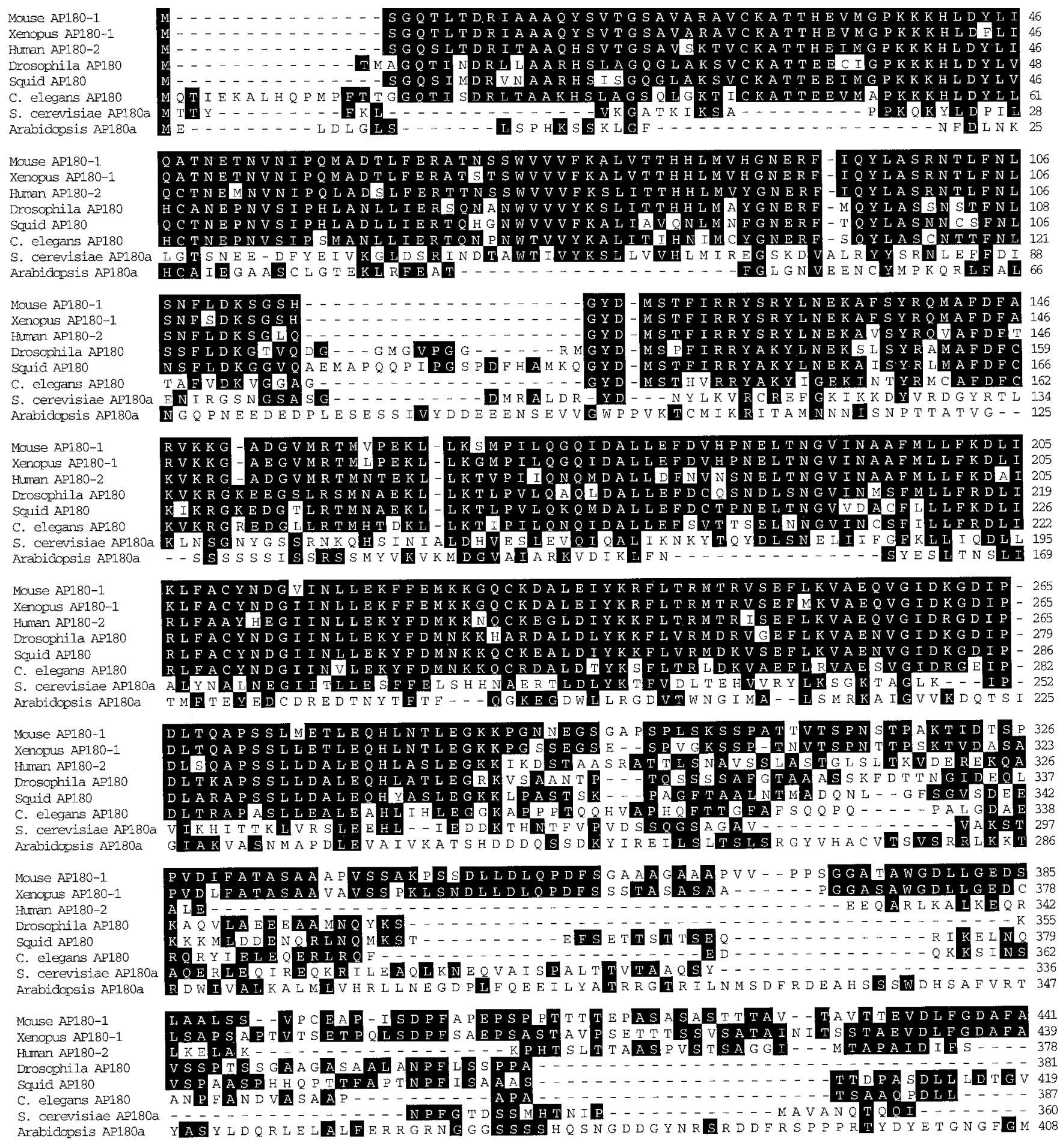

Figure 1. Amino acid sequence alignment of the AP180 family members. Residues present in two or more family members are highlighted in black.

the protein. In addition, chronic genetic manipulations may allow developmental or compensatory effects that could indirectly affect endocytosis.

We find that treatments that acutely perturb clathrin assembly in vitro affect synaptic transmission in vivo. In particular, blocking clathrin assembly by AP180 in vivo rapidly blocks synaptic vesicle endocytosis, similar to what was observed by the genetic mutation of AP180. Our results indicate that clathrin and its assembly by AP180 play predominant roles in synaptic vesicle endocytosis.

\section{MATERIALS AND METHODS}

Cloning of squid AP180. A $\lambda$ gt10 cDNA library prepared from squid stellate ganglion (kindly provided by Dr. James Battey, National Institutes of Health, Bethesda, MD) was screened by low-stringency hybridization with a mouse AP180 cDNA probe corresponding to nucleotides 504-1408 (amino acids 168-469) (Zhou et al., 1992). Nitrocellulose membranes were hybridized with $\alpha-{ }^{32} \mathrm{P}-\mathrm{dCTP}$-labeled mouse AP180 cDNA probe at $50^{\circ} \mathrm{C}$ overnight in hybridization solution $(6 \times \mathrm{SSC}, 5 \times$ Denhardt's, $0.1 \%$ SDS, and $100 \mu \mathrm{g} / \mathrm{ml}$ of denatured salmon sperm 
Mouse AP180-1 Xenopus AP180-1 Human AP180-2 Drosophila AP180 Squid AP180

C. elegans AP180 S. cerevisiae AP180 Arabidopsis AP180a

Mouse AP180-1 Xenopus AP180-1

Euman AP180-2

Drosophila AP180

Squid AP180

C. elegans AP180

S. cerevisiae AP180a Arabidopsis AP180a

Mouse AP180-1

Xemopus AP180-1

Fiman AP180-2

Drosophila AP180

Squid AP180

C. elegans AP180

S. cerevisiae AP180a

Arabidopsis AP180a

Mouse AP180-1

Xenopus AP180-1

Furnan AP180-2

Drosophila AP180

Squid AP180

C. elegans AP180

S. cerevisiae AP180a

Arabidopsis AP180a

Mouse AP180-1

Xenopus AP180-1

Human AP180-2

Drosophila AP180

souid AP180

C. elegans AP180

S. cerevisiae AP180

Arabidopsis AP180a

Mouse AP180-1

Xenopus AP180-1

Human AP1 80-2

Drosophila AP180

Squid AP180

C. elegans AP180

S. cerevisiae AP180

Arabidopsis AP180a

Mouse AP180-1

Xenopus AP180-1

Humar AP180-2

Drosophila AP180

Squid AP180

C. elegans AP180

S. cerevisiae AP180a

Arabidopsis AP180a

Mouse AP180-1

Xenopus AP180-1.

filman AP180-2

Drosophila AP180

Squid AP180

C. elegans AP180

S. cerevisiae AP18

Arabidopsis AP180a
ASPGEAPAASE GATAPATPAPVAAALDACSGNDPFAPSEGSAEAAPELDLFAMK P E TSAP 502

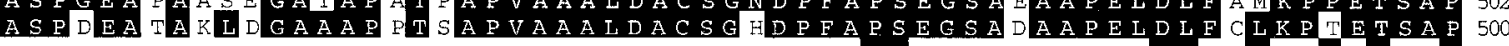

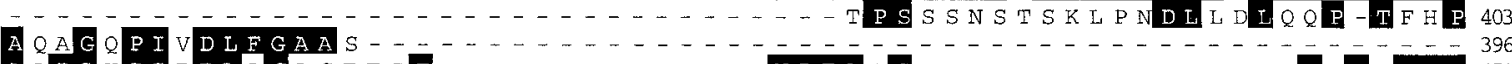

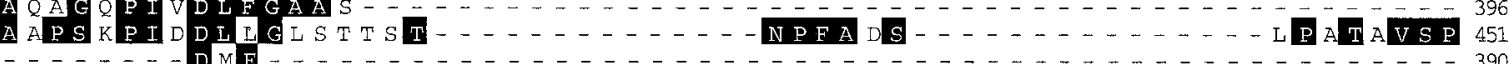

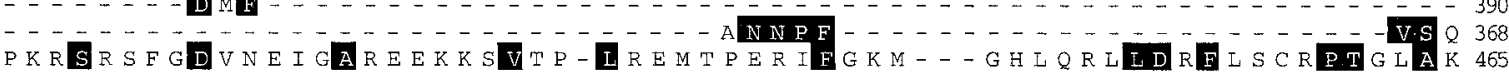

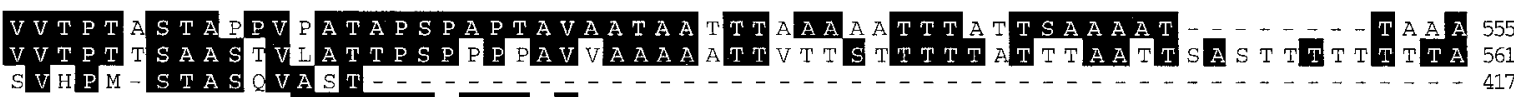

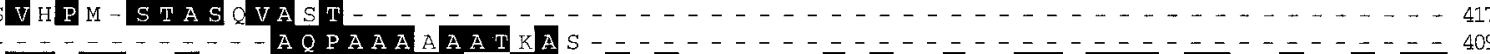

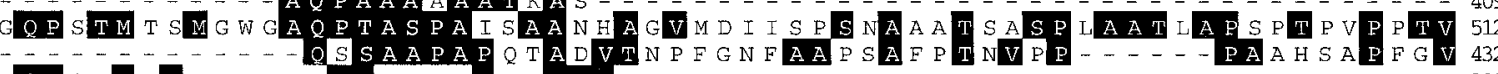

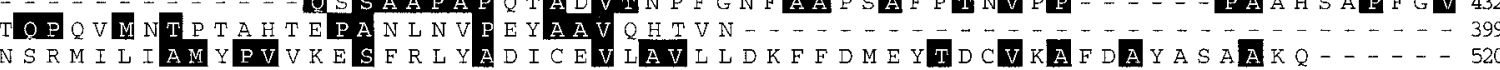

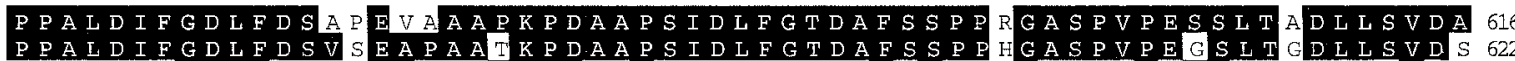

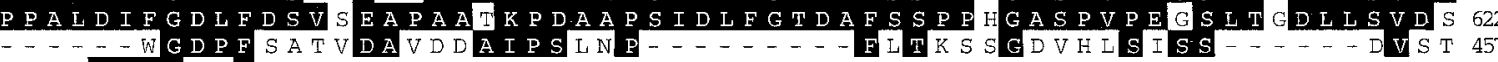

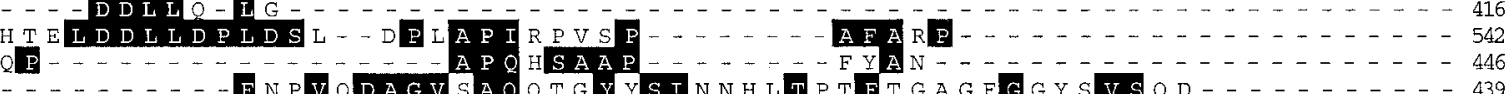
IDEIIAFYHWCKDTGVA-RSSEYDEVQRITSKLLETLEEFVRDRAKRAKSPERKEIE 577
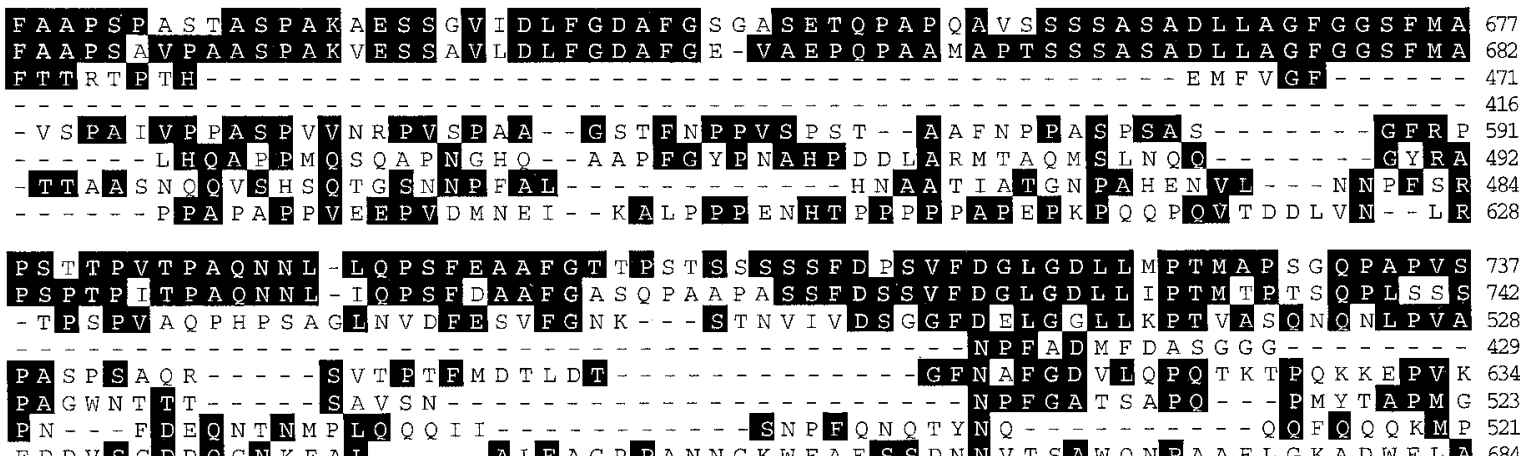

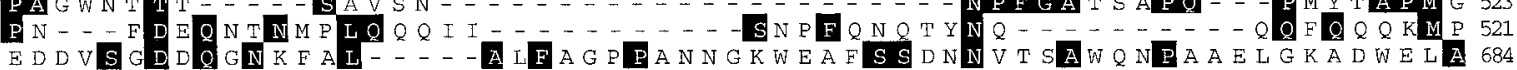

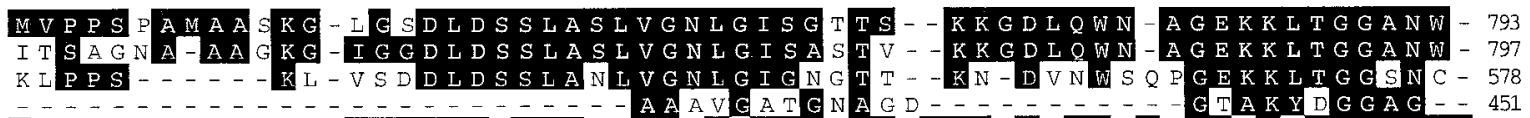

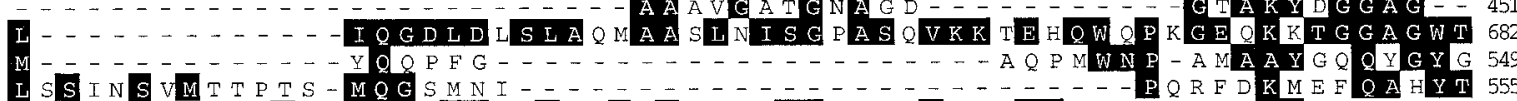

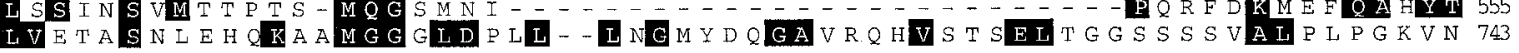

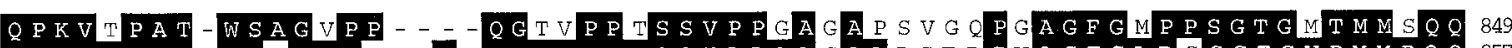

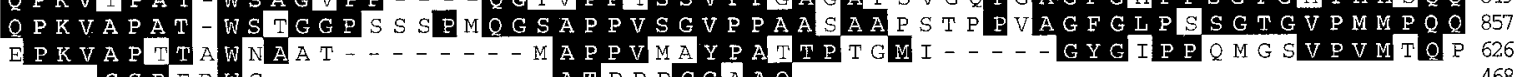

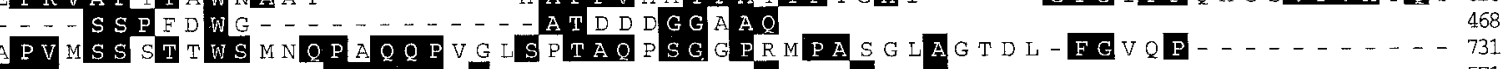
QPV-......

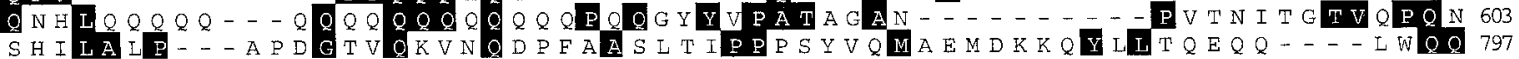

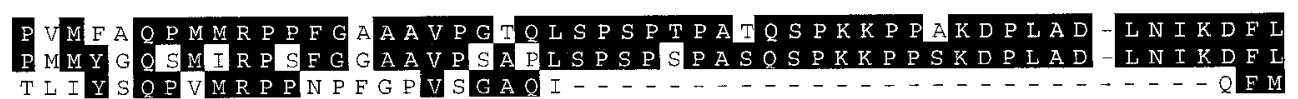

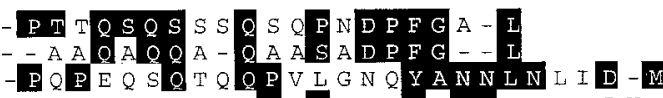

FY Y OQ

Figure 1 Continued
DNA). The filters were washed extensively at room temperature in $2 \times$ $\mathrm{SSC} / 0.1 \% \mathrm{SDS}$ for $20 \mathrm{~min}$ and then at $50^{\circ} \mathrm{C}$ in $1 \times \mathrm{SSC} / 0.1 \% \mathrm{SDS}$ for 1 $\mathrm{hr}$. The positive clones were plaque-purified and subcloned into pBluescript vector $\mathrm{KS}^{+}$at the EcoRI site. Single-stranded DNA was prepared from each clone and sequenced by using the dideoxy chain termination method with Sequenase Version 2.0 DNA Sequencing kit (United States Biochemicals, Cleveland, OH). A 1082 base pair (bp) long clone was isolated that was homologous to mouse AP180.

This clone was used to rescreen the squid stellate ganglion cDNA library under high-stringency hybridization conditions. Nitrocellulose filters were hybridized at $65^{\circ} \mathrm{C}$ overnight. Next the filters were washed extensively in $1 \times \mathrm{SSC} / 0.1 \% \mathrm{SDS}$ at room temperature for $1 \mathrm{hr}$, followed by a $30 \mathrm{~min}$ wash at $60^{\circ} \mathrm{C}$. The positive clones were subcloned into pBluescript vector $\mathrm{KS}^{+}$at the EcoRI site for sequencing. Of the 36 clones identified, the longest clone of $2575 \mathrm{bp}$ contained the entire open reading frame. Basic Local Alignment Search Tool (BLAST) searches of the National Center for Biotechnology Information (Bethesda, MD) nonredundant protein database revealed that the sequence is homologous to a number of AP180-related entries in the database.

Analysis of the squid AP180 sequence. Multiple sequence alignments were generated with Megalign running under the Lasergene software package (DNAstar, Madison, WI), using the Clustal method (Thompson 
et al., 1994) and the PAM250 residue weight table. The alignment displayed in Figure 1 included the following sequences, with the indicated accession numbers: mouse AP180-1 (2492687) (Zhou et al., 1992), Xenopus AP180-1 (AF182340) (Zhou, 1994), human AP180-2 (1373146) (Dreyling et al., 1996), Drosophila AP180 (4160434) (Zhang et al., 1998), Caenorhabditis elegans AP180 (AF144257) (Nonet et al., 1999), Saccharomyces cerevisiae AP180a (731735) (Wendland and Emr, 1998), and Arabidopsis AP180a (2864615). The following sequences were added to the multiple sequence alignment for the construction of the phylogeny displayed in Figure 2: human AP180-1 (3327126) (Ishikawa et al., 1998), rat AP180-1 (2492686) (Morris et al., 1993), rat AP180-2 (2792502), S. cerevisiae AP180b (1723758) (Wendland and Emr, 1998), and S. pombe AP180 (3150136). Two types of phylogenetic analysis were performed, maximum parsimony (Swofford, 1998) and neighbor joining (Felsenstein, 1985). Both were performed with PAUP4.0b2 (Swofford, 1998). Robustness of the results was assessed by bootstrap analysis. One thousand bootstrap replications, with five random additions each, were done by using unweighted parsimony. One thousand bootstrap replications were done by using neighbor joining, with tree-bisection reconnection branch swapping to define the shortest tree. The results of the two analyses were in agreement, although the level of support for particular nodes differed. The tree was rooted by using the most divergent sequence, Arabidopsis AP180a, as the out-group (data not shown). Bootstrap values are shown above each node (parsimony/neighbor joining). The reported pairwise identities (see Table 1 and text) were calculated by dividing the number of positions with identical residues in the multiple sequence alignment (see Fig. 1) by the shortest of the two sequences being compared. The 33 $\mathrm{kDa}$ N-terminal domain of mouse AP180 already has been defined as comprising amino acids $1-304$, and the $58 \mathrm{kDa} \mathrm{C}$-terminal domain of mouse AP180 has been defined as comprising amino acids 305-901 (Ye and Lafer, 1995a,b). For each of the AP180 family members that was analyzed, the last residue of the N-terminal domain was taken to be the residue that aligned with amino acid 304 of mouse AP180.

Expression of recombinant AP180. The entire open reading frame of squid AP180 was amplified by reverse transcription PCR. Total RNA from squid optic lobe was isolated with Trizol (Life Technologies, Gaithersburg, MD) and reverse-transcribed into cDNA, using SuperScript II RT (Life Technologies). The synthesized first-strand cDNA was used as a template for a PCR reaction that used sense primer (5'AGCGAGCTCCCCGGGATGTCTGGACAGAGTATAATG-3') and antisense primer (5'-GCTCTCGAGCCCGGGTCACAAAGCACCA AATGGATC-3'). An $X b a I$ site flanking the open reading frame was introduced into both PCR primers. Pfu DNA polymerase was used for the PCR reaction, following the protocol provided by Stratagene (La Jolla, CA). The PCR product was subcloned into pGEX-X at the $X b a \mathrm{I}$ site. To amplify the $45 \mathrm{kDa}$ putative clathrin assembly domain of squid AP180, we performed PCR, using the full-length AP180 as a template. A sense primer (5'-AGCGAGCTCCCGGGTTCAACATCTAATGGTGTGTCA-3') that contains a SmaI site and an antisense primer (5'GCT CCCGGGCTCGAGTCACAAAGCACCAAATGGATC-3') that contains a $X h o I$ site were used for the PCR reaction. The PCR product was subcloned into pGEX-4T-1 at the SmaI and XhoI sites. The sequence of the full-length squid AP180 insert was confirmed further by sequencing and was found to be identical to that of the cDNA clone reported in Figure 1. Protein was expressed and purified from suitably transformed BL21 cells as previously described for mouse AP180 (Zhou et al., 1993).

Clathrin assembly. The assembly of bovine brain clathrin into coats was measured by ultracentrifugation in the presence of recombinant proteins, consisting of GST fused to bovine AP180 (bAP180), squid GSTAP180 (sAP180), or the $45 \mathrm{kDa}$ C-terminal domain of squid AP180 (sC45) (Hao et al., 1997). In this assay the coats that have a high sedimentation coefficient are pelleted, whereas soluble clathrin triskelia with low sedimentation coefficient remain in the supernatant. Briefly, all proteins first were dialyzed overnight into $10 \mathrm{~mm}$ Tris- $\mathrm{HCl}, \mathrm{pH} 8.5$, at $4^{\circ} \mathrm{C}$. Individually, bAP180, sAP180, sC45, or GST was combined with bovine clathrin, and the assembly reaction was initiated by adding 1:10 volume of $1 \mathrm{M}$ MES-NaOH, $\mathrm{pH}$ 6.5. The final conditions in the reaction were $0.8 \mu \mathrm{M}$ clathrin, $0.1 \mathrm{M}$ MES-NaOH, $9 \mathrm{~mm}$ Tris-HCl, $\mathrm{pH} 6.5$, and the indicated concentrations of assembly proteins in a volume of $200 \mu \mathrm{l}$. The mixture was incubated on ice for $60 \mathrm{~min}$ and then was centrifuged at $400,000 \times g, 4^{\circ} \mathrm{C}$ for $6 \mathrm{~min}$. The upper $80 \%$ of the supernatant was removed and analyzed by SDS-PAGE and Coomassie blue staining. The percentage of clathrin that was assembled was determined by the relative depletion of clathrin from the solutions before and after centrifugation. This was quantified by densitometry of Coomassie blue-stained gels
(Molecular Dynamics Personal Densitometer SI, Sunnyvale, CA), using Image Quant software. In the absence of assembly proteins, $\sim 5 \%$ of the clathrin sedimented. This percentage was treated as background clathrin assembly and was subtracted from all of the data points. Morphology of the clathrin cages was assessed by electron microscopy (see Fig. $3 E$ ), as described previously (Ye and Lafer, 1995a). Assembled clathrin coat structures were applied to freshly glow-discharged Formvar carboncoated grids and negatively stained with $1 \%$ uranyl acetate. The specimens were viewed in a JEOL 1200 EX Transmission electron microscope at a magnification of $40,000 \times$.

Inhibition of clathrin assembly by peptides. Peptides from the clathrin assembly domain (sC45) of squid AP180 were synthesized as potential inhibitors of clathrin assembly in vitro. The sequences of the peptides used in this study are as follows: NGVSDEEKKKMLDDENQRLNQ (s180 pep); KSLGEDDNRNMVEEDKNQLQK (Scram s180 pep1); QNEDSMKDVQKNLENLKGDRE (Scram s180 pep2). To test the ability of these peptides to inhibit clathrin assembly, we made $5 \mathrm{~mm}$ stock solutions in $10 \mathrm{~mm}$ Tris- $\mathrm{HCl}, \mathrm{pH}$ 8.5. The $\mathrm{pH}$ of the solution was adjusted by using $2 \mathrm{M} \mathrm{NaOH}$ when required. Bovine clathrin and sAP180 were combined in $10 \mathrm{~mm}$ Tris- $\mathrm{HCl}, \mathrm{pH} 8.5$, to which an appropriate amount of the peptide solution was added. The assembly was initiated by the addition of 1:10 volume of $1 \mathrm{M}$ MES-NaOH, $\mathrm{pH}$ 6.7. The final conditions in the reaction mixture were $0.5 \mu \mathrm{M}$ clathrin, $1.5-2.0 \mu \mathrm{M}$ sAP180, $0.1 \mathrm{M}$ MES-NaOH, pH 6.7, and 0-1000 $\mu$ M peptide in a final volume of $200 \mu \mathrm{l}$. The mixture was incubated on ice for $45 \mathrm{~min}$, followed by centrifugation at $400,000 \times g$, at $4^{\circ} \mathrm{C}$ for $6 \mathrm{~min}$. The amount of clathrin that was assembled was determined as described above. Clathrin assembled in the presence of sAP180, but without peptides, served as $100 \%$ assembly. The inhibition by the peptides was calculated as the relative amount of assembly in the presence of the peptides as compared with the assembly in the absence of the peptides. Peptides were synthesized by Dr. Lynda Bonewald at the Protein Core Facility of the University of Texas Health Science Center (San Antonio, TX).

Electrophysiological analysis of synaptic transmission. Electrical measurements were made on giant synapses in isolated stellate ganglia of the squid, Loligo pealei, as described in Bommert et al., (1993). Ganglia were superfused with oxygenated physiological saline $\left(10-15^{\circ} \mathrm{C}\right)$ containing (in mM) $466 \mathrm{NaCl}, 54 \mathrm{MgCl}_{2}, 11 \mathrm{CaCl}_{2}, 10 \mathrm{KCl}, 3 \mathrm{NaHCO}_{3}$, and 10 HEPES, pH 7.2. Electrodes filled with $3 \mathrm{M} \mathrm{KCl}$ were inserted into the presynaptic and postsynaptic axons. Action potentials were evoked every $30 \mathrm{sec}(0.033 \mathrm{~Hz})$ by depolarizing the presynaptic axon with a current pulse (0.7-1.9 $\mu \mathrm{A}, 0.5 \mathrm{msec}$ duration). Transmitter release was measured by recording postsynaptic potentials (PSPs) elicited by the presynaptic action potentials. Signals were recorded with an Axoclamp-2A amplifier (Axon Instruments, Foster City, CA) and acquired and analyzed with Axobasic programs written by F. Schweizer (UCLA, Los Angeles, CA).

The mammalian proteins used for microinjection were expressed and purified as described by Ye and Lafer (1995b). The most biologically abundant isoform of mouse AP180 (AS108 ${ }^{+} \mathrm{AS} 15^{-}$) was used (Zhou et al., 1993). All microinjected peptides and proteins were dissolved in an injection solution containing (in $\mathrm{mM}$ ) $250 \mathrm{~K}$-isothionate, $200 \mathrm{KCl}, 100$ taurine, and 50 HEPES, pH-adjusted to 7.4 with $\mathrm{NaOH}$. These solutions were microinjected into the giant presynaptic terminal via a third electrode connected to a Picospritzer injector (General Valve, NJ) that applied pulses of positive pressure (10-80 msec; $10-100$ psi; $\mathrm{N}_{2}$ gas). FITC-dextran ( 3 kDa MW; $100 \mu \mathrm{M}$; Molecular Probes, Eugene, OR) was coinjected to estimate the amount of protein or peptide that had been injected. The resulting fluorescence was imaged with a Zeiss Axioskop microscope $(10 \times$ magnification, 0.25 numerical aperture objective) and detected with a Cohu SIT camera. Fluorescence images were processed with Image-1 software (Universal Imaging, Philadelphia, PA).

Electron microscopic analysis. Terminals were fixed for electron microscopy with $2.5 \%$ glutaraldehyde and processed as described in Sanchez et al. (1990). When active peptides were injected, the terminals were fixed after synaptic transmission was inhibited by $80 \%$ or more. Fixed terminals were sectioned ( $80 \mathrm{~nm}$ thickness) at intervals of 50 or $75 \mu \mathrm{m}$ through the entire length of the synaptic terminal. Sections were examined with a JEM-1200 ExII (JOEL USA, Peabody, MA) electron microscope, and images were digitized and analyzed with Image-1 software. Terminal structure was quantified as described previously (Hess et al., 1993; Burns et al., 1998). Because active zones typically are spaced $\sim 1 \mu \mathrm{m}$ apart, only synaptic vesicles within $500 \mathrm{~nm}$ of the active zone were measured to ensure that each vesicle was counted only once.

Membrane area was calculated for each section by measuring the perimeter of the presynaptic terminal in low magnification $(500 \times)$ im- 
$\overline{\text { Table 1. Sequence identities between squid AP180 and the other AP180 }}$ family members

\begin{tabular}{llll} 
& \multicolumn{3}{l}{ Identity to squid AP180 $(\%)$} \\
\cline { 2 - 4 } & $\begin{array}{l}\text { Whole } \\
\text { protein }\end{array}$ & $\begin{array}{l}\text { N-terminal } \\
\text { domain }\end{array}$ & $\begin{array}{l}\text { C-terminal } \\
\text { domain }\end{array}$ \\
\hline Mouse AP180 1 & 40 & 66 & 24 \\
Xenopus AP180 1 & 41 & 66 & 26 \\
Human AP180 2 & 39 & 70 & 13 \\
Drosophila AP180 & 61 & 74 & 35 \\
C. elegans AP180 & 46 & 64 & 26 \\
S. cerevisiae AP180a & 17 & 24 & 12 \\
Arabidopsis AP180a & 11 & 16 & 9 \\
\hline
\end{tabular}

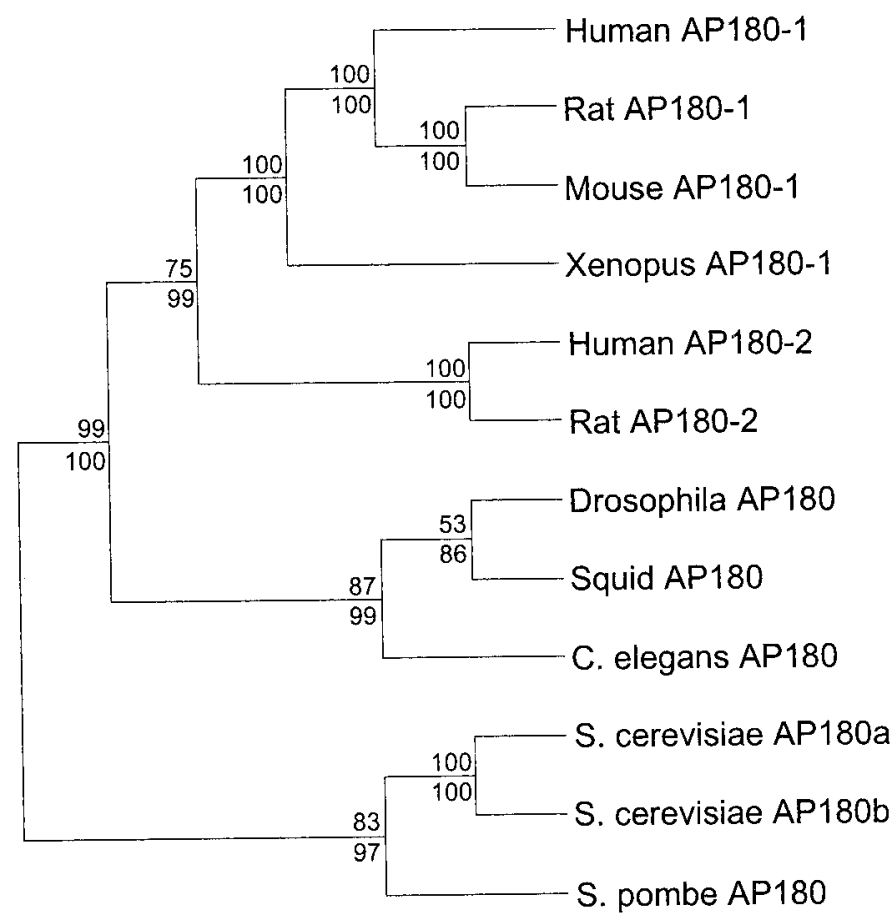

Figure 2. Phylogenetic relationships among the AP180 family members.

ages. Such measurements probably represent underestimates because of small plasma membrane invaginations that could not be detected at low magnification. To determine the magnitude of this effect, we measured actual plasma membrane distance from selected regions in highmagnification images $(12,000 \times)$. These measurements indicated that the plasma membrane perimeter of terminals injected with s180 peptide was $36 \%$ larger than estimated from the low-magnification images, whereas the control terminals were underestimated by $30 \%$. Then the perimeter measurements made at low magnification were increased by these amounts. Plasma membrane perimeter was multiplied by the thickness of the section $(80 \mathrm{~nm})$ to calculate the surface area of plasma membrane in each section. The surface area of synaptic and coated vesicles was calculated as $4 \pi r^{2}$, where $r$ is the measured radius of these vesicles. The surface area per vesicle was multiplied by the mean number of vesicles per active zone, yielding the amount of vesicle area per active zone, and then was multiplied by the number of active zones per section to yield the mean surface area in synaptic and coated vesicles in each section.

\section{RESULTS}

\section{Cloning of the squid homolog of AP180}

Low-stringency screening of a squid $\lambda \mathrm{gt10}$ cDNA library, by hybridization with a mouse AP180 cDNA probe, identified a 1082 bp clone that had high homology to mouse AP180. This clone was used to rescreen the cDNA library by high-stringency hybridization and identified 36 additional clones. The sequence of the longest clone was 2575 nucleotides, and it covered 90 nucleotides of the $5^{\prime}$ untranslated region, the entire coding region, and the entire $3^{\prime}$ untranslated region to the $\operatorname{poly}\left(\mathrm{A}^{+}\right)$tail. It contained a single open reading frame with two upstream in-frame stop codons. The open reading frame of squid AP180 encoded a 751 amino acid protein with a predicted molecular weight of 80,200 Da.

The sequence of squid AP180 is homologous to a number of AP180-related entries in the protein database of the National Center for Biotechnology Information. Because several of these entries came from genome-sequencing projects and the predicted proteins have not yet been named, we devised a uniform nomenclature for the AP180 family members. Mouse AP180, the first vertebrate AP180 to be cloned and sequenced, was assigned the name AP180-1. All vertebrate sequences highly homologous to mouse AP180-1 also were assigned the name AP180-1. Mouse AP180-1 mRNA is restricted to neuronal cells, and its protein is synapse-specific (Perry et al., 1991, 1992; Sousa et al., 1992); this may be the case for the other members of the AP180-1 group. A second vertebrate AP180-related gene with $48 \%$ identity to AP180-1 was isolated from a human myeloid leukemia cell line and was called clathrin assembly lymphoid myeloid leukemia gene, or CALM (Dreyling et al., 1996). We assigned the name AP180-2 to human CALM as well as to all vertebrate sequences highly homologous to human AP180-2. AP180-2 mRNA is expressed ubiquitously in a wide range of human cell types, and this may be true for other members of the AP180-2 group (Dreyling et al., 1996). The AP180related genes of nonvertebrates are called AP180, with multiple genes distinguished by letters according to the precedent set by the S. cerevisiae AP180 genes (Wendland and Emr, 1998).

The relationship between squid AP180 and other representatives of the AP180 family was revealed by a multiple sequence alignment (Fig. 1). When more than one alternatively spliced isoform has been reported for a family member, we used the longest available isoform in constructing the alignment. Gaps in the alignment reflect the diverse range of sizes of the AP180 family members, especially within the C-terminal domain. The smallest reported family member is Drosophila AP180, which has 469 amino acids (Zhang et al., 1998). The largest reported family member is rat AP180, which has 915 amino acids (Morris et al., 1993). Examination of the alignment reveals a protein family that is much better conserved in the $\sim 33 \mathrm{kDa} \mathrm{N}$-terminal domain than in the variably sized C-terminal domain (Fig. 1). This observation is supported by an analysis of the pairwise identity scores of the aligned sequences (Table 1). For example, whereas the overall identity between squid and mouse AP180 is $40 \%$, the identity between their N-terminal domains is $66 \%$, and the identity between their C-terminal domains is $24 \%$.

Phylogenetic analyses of all of the available AP180 family members reveal that squid AP180 is related most closely to the other invertebrate AP180s, with the strongest similarity to Drosophila AP180 (Fig. 2). To our surprise, the mammalian AP180-2 subfamily is more closely related to the invertebrate AP180s than it is to the mammalian AP180-1 subfamily. This indicates that the AP180-2 subfamily appeared before the AP180-1 subfamily during evolution.

\section{Biochemical characterization of squid AP180 and its C-terminal domain}

The clathrin assembly activity of mouse AP180-1 resides within its C-terminal domain (Ye and Lafer, 1995b), which our analysis 

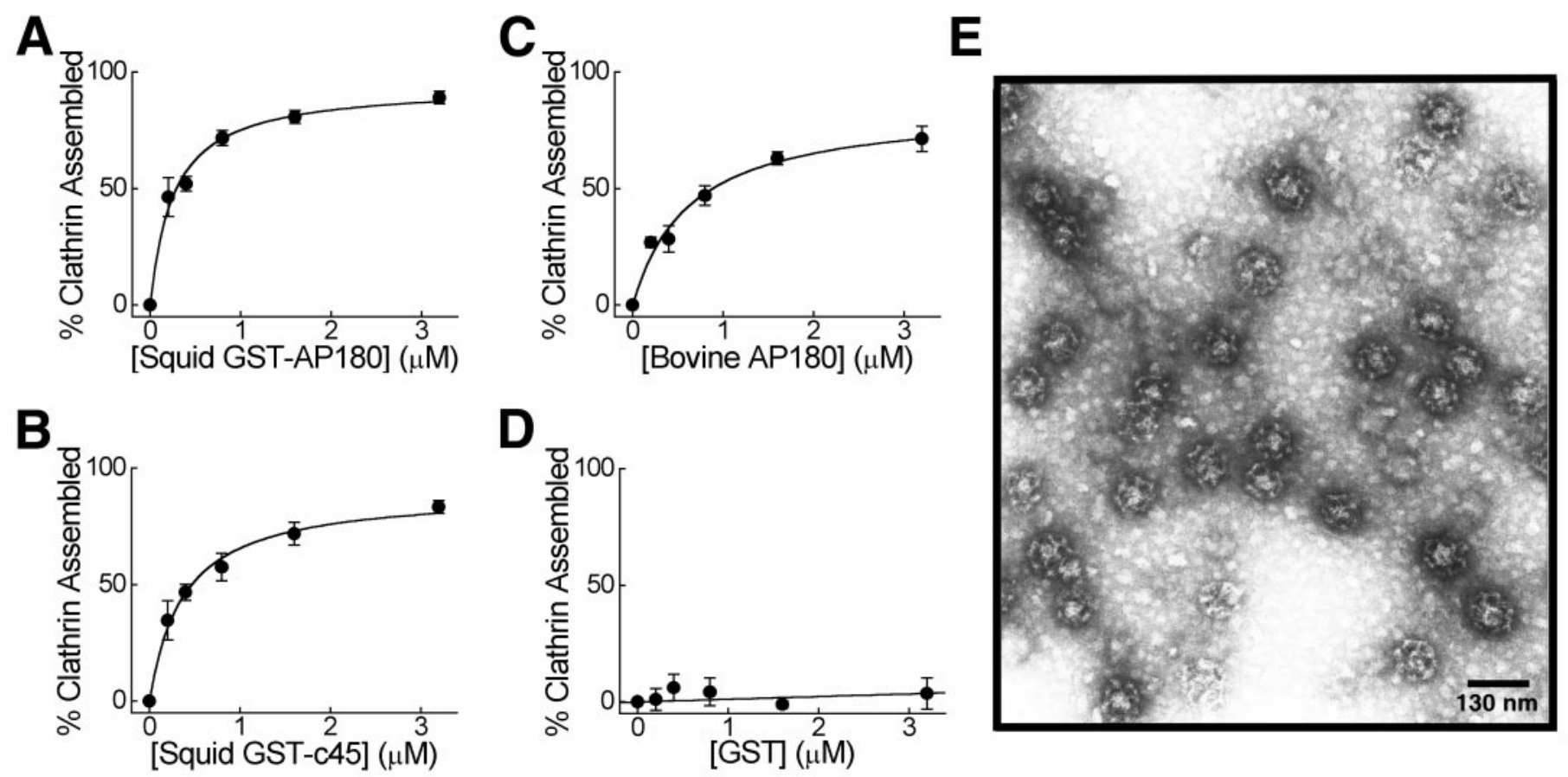

Figure 3. Squid GST-AP180 and squid GST-c45, but not GST, assemble clathrin as efficiently as bovine AP180. $A-D$, Clathrin assembly by squid GST-AP180 $(A)$, squid GST-c45 $(B)$, bovine AP180 $(C)$, and GST $(D)$. Points represent the mean of three to four independent experiments, and error bars indicate the SEM values. Half-maximal concentrations for clathrin assembly, determined by fits to a rectangular hyperbola function (solid lines), were $0.3 \mu \mathrm{M}$ for squid GST-AP180, $0.4 \mu \mathrm{M}$ for GST-c45, and $0.6 \mu \mathrm{M}$ for bovine AP180. The maximum amount of assembly ranged from 85 to $94 \%$ for these three proteins. $E$, Electron micrograph of clathrin coats assembled by squid GST-AP180.

indicates is not well conserved among other members of the AP180 family (see Fig. 1). Thus, it is possible that other AP180 forms do not assemble clathrin. For this reason we asked whether squid AP180 can assemble clathrin. To study the functional properties of squid AP180 in vitro, we expressed in bacteria glutathione $S$-transferase (GST) fusions of full-length squid AP180 (aa 1-751) (sAP180) and its C-terminal domain (aa 313751) (sC45). Because squid AP180 is shorter than mouse AP180, the amino acids that correspond to the $58 \mathrm{kDa}$ clathrin assembly domain of mouse AP180 (mC58; Ye and Lafer, 1995b) encode a $45 \mathrm{kDa}$ protein in squid. The abilities of sAP180, sC45, GST, and bovine AP180 (bAP180) to assemble bovine clathrin were compared in an in vitro assay that measured the incorporation of clathrin triskelia into cages (Fig. 3). Both sAP180 and sC45 were

\begin{tabular}{|c|c|c|}
\hline AP180 reagent & Number of injections & Mean effect (SEM) (\%) \\
\hline mAP180 & 9 & $8.1(4.0)$ \\
\hline $\mathrm{mC} 58$ & 13 & $17.9(6.5)$ \\
\hline mM42 & 4 & $5.8(7.1)$ \\
\hline mN33 & 2 & $5.1(5.1)$ \\
\hline sAP180 & 8 & $3.8(7.6)$ \\
\hline sC45 & 10 & $16.6(9.2)$ \\
\hline GST & 10 & $5.1(4.1)$ \\
\hline Carrier solution & 13 & $-0.1(2.3)$ \\
\hline s180 pep & 17 & $-35.4(7.2)$ \\
\hline Scram s180 pep1 & 2 & $-3.8(2.4)$ \\
\hline
\end{tabular}

able to assemble bovine clathrin in a concentration-dependent manner (Fig. 3A,B). In terms of the concentrations required for clathrin assembly, as well as the maximal amount of assembly, both squid constructs were very similar to bovine AP180 (Fig. $3 C$ ). GST alone did not assemble clathrin (Fig. 3D), showing that the clathrin assembly activity of the fusions was attributable to the AP180 rather than to the GST. The cages assembled by sAP180 were homogeneous in size (Fig. 3E), as has been reported for mammalian AP180s (Ye and Lafer, 1995a). These results indicate that squid AP180, like its mammalian homologs, is a clathrin assembly protein. Further, the ability to assemble clathrin resides in the $45 \mathrm{kDa}$ C-terminal domain of squid AP180, which indicates that enough tertiary structure is conserved to mediate clathrin assembly despite the divergent primary sequences in the C-terminal domains of mammalian and squid AP180.

\section{The clathrin assembly domain of AP180 enhances synaptic transmission}

To determine whether clathrin assembly by AP180 is important for synaptic vesicle endocytosis, we microinjected various forms of AP180 into the squid giant presynaptic terminal to determine their actions on neurotransmitter release. If AP180 stimulates clathrin assembly in vivo and clathrin assembly is essential for synaptic vesicle endocytosis, then injecting AP180 might enhance synaptic transmission. Synaptic transmission was evoked by single action potentials elicited every $30 \mathrm{sec}(0.03 \mathrm{~Hz})$ by currents injected directly into the presynaptic axon, and postsynaptic responses were recorded during the injection of AP180 reagents. Although full-length mouse GST-AP180 (mAP180) and sAP180 had no effect on synaptic transmission (Table 2), the injection of 


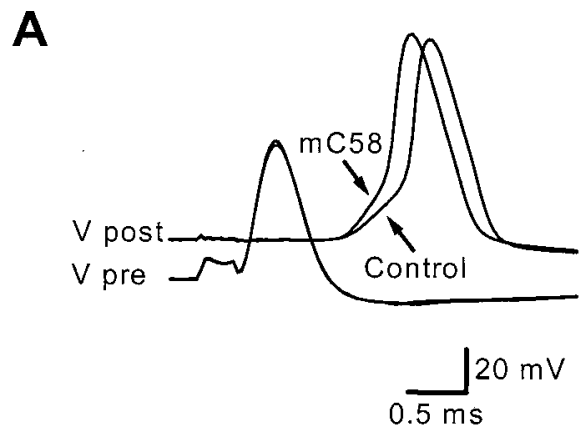

B

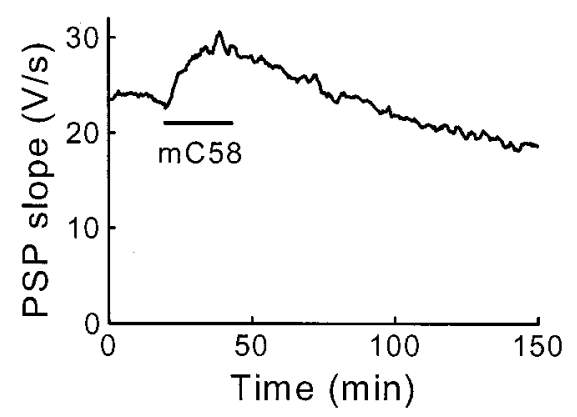

C

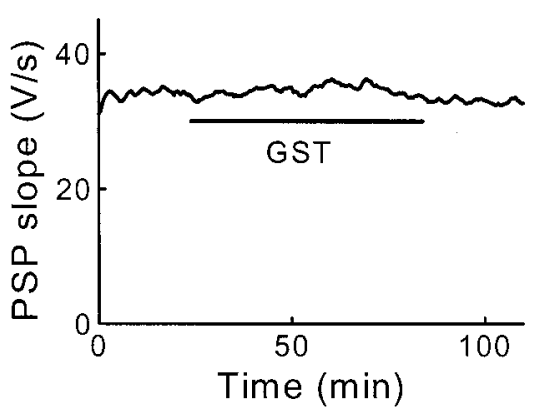

Figure 4. Presynaptic microinjection of the $58 \mathrm{kDa}$ clathrin assembly domain of mouse AP180-1 ( $m C 58)$ enhances transmission. A, Superimposed traces of presynaptic $\left(V_{\text {pre }}\right)$ and postsynaptic $\left(V_{\text {post }}\right)$ responses during microinjection of mC58. mC58 enhanced the postsynaptic responses, whereas the presynaptic response remained unchanged. $B$, Time course of response to the injection of mC58 $(10 \mu \mathrm{M}$; during bar $)$. mC58 reversibly enhanced transmitter release when the synapse was stimulated at $0.03 \mathrm{~Hz}$. C, GST alone had no effect on transmitter release.

the clathrin assembly domains of either squid (sC45) or mouse AP180 (mC58) enhanced PSPs produced by presynaptic action potentials (Fig. 4A,B, Table 2). Injected mC58 enhanced transmitter release without altering the presynaptic action potential (Fig. 4A), indicating that action potential generation was unaffected by the protein. The enhancement of the postsynaptic response was reversible over time (Fig. $4 B$ ), presumably attributable to mC58 diffusing out of the terminal and into the rest of the presynaptic neuron (Bommert et al., 1993; Burns et al., 1998). This indicates that the inhibition was attributable to the injected protein rather than to some irreversible consequence of the injection procedure. Control injections of GST also had no effect on synaptic transmission, further documenting the specificity of the injected proteins (Fig. 4C, Table 2).

The lack of effect of full-length AP180 could indicate that the microinjected protein was inhibited by regulatory factors. Potential regulatory factors include endogenous inositides, which inhibit AP180-mediated clathrin assembly by binding to the regulatory N-terminal domain of AP180 (Ye et al., 1995; Hao et al.,
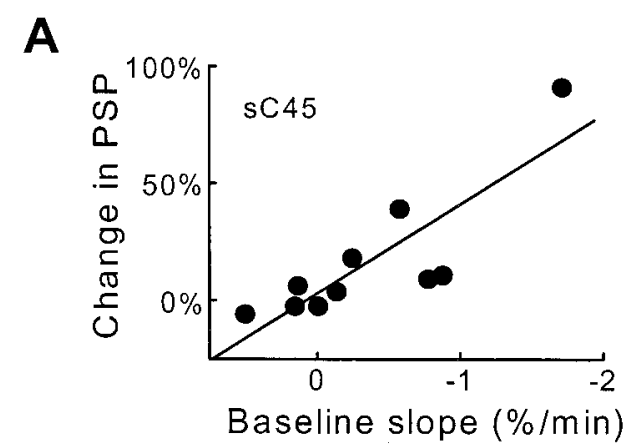

B

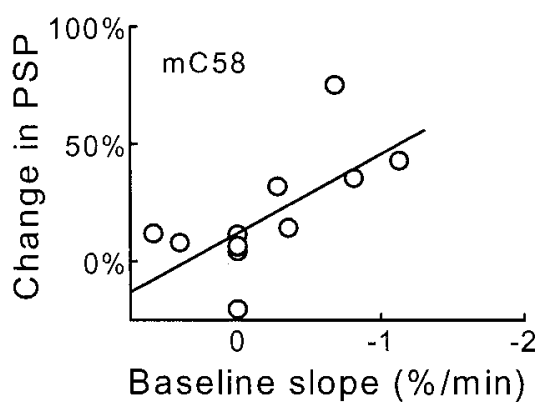

C

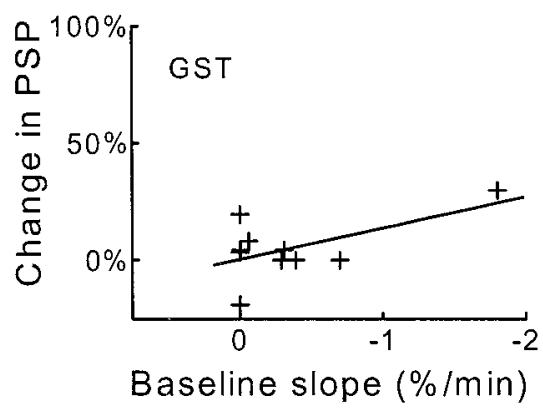

Figure 5. The effects of sC45 and mC58 depend on the physiological state of the synapse. $A, B$, When basal synaptic transmission (Baseline slope, measured as the rate of change of the PSP slope) was declining, the resulting enhancement in transmission (Change in PSP) seen with $\mathrm{sC} 45$ $(A)$ and mC58 $(B)$ is larger than when there is little or no decline in basal synaptic transmission. $C$, Injection of GST had little or no effect, even when basal synaptic transmission was declining rapidly. Data were fit by a linear function (solid lines).

1997). If this is the case, then injecting the N-terminal domainwhich has no clathrin assembly activity in vitro (Ye and Lafer, 1995a,b) - should not affect synaptic transmission. To test this possibility, we injected the $33 \mathrm{kDa}$-terminal domain of mouse AP180 (mN33) and found that this domain indeed had no effect on transmitter release (Table 2). Likewise, the central $42 \mathrm{kDa}$ domain of mouse AP180 (mM42), which also has no clathrin assembly activity in vitro (Ye and Lafer, 1995b), did not affect synaptic transmission (Table 2). These results suggest that increasing the amount of clathrin assembly in vivo by AP180 enhances neurotransmitter release and that this clathrin assembly is regulated by the $\mathrm{N}$-terminal domain of the protein.

The stimulatory actions of injected sC45 and mC58 were sensitive to the physiological state of the presynaptic terminal (Fig. 5). During the course of our experiments, occasionally synapses were encountered in which synaptic transmission was declining. In these cases, injecting $\mathrm{sC} 45$ or mC58 caused a large enhancement of synaptic transmission, but a much smaller effect was 


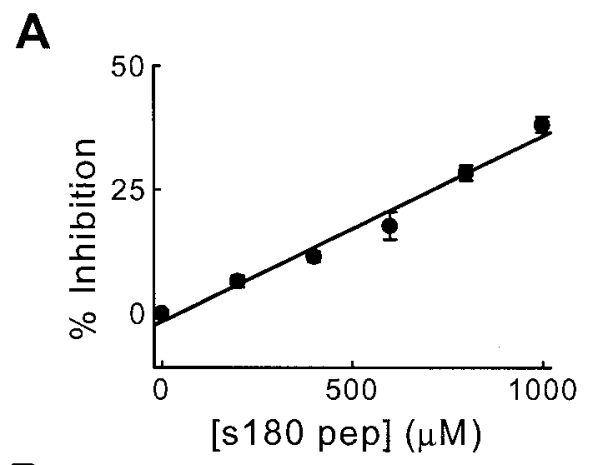

B

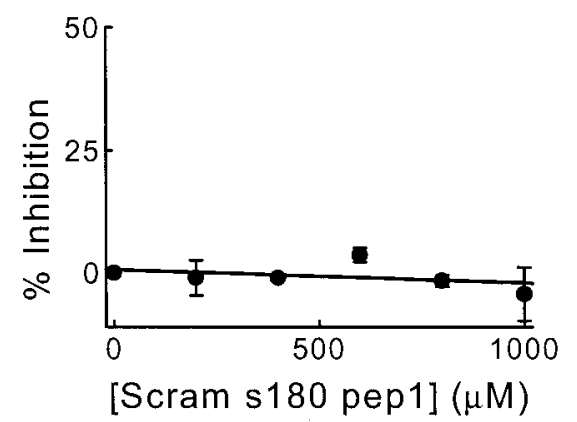

C

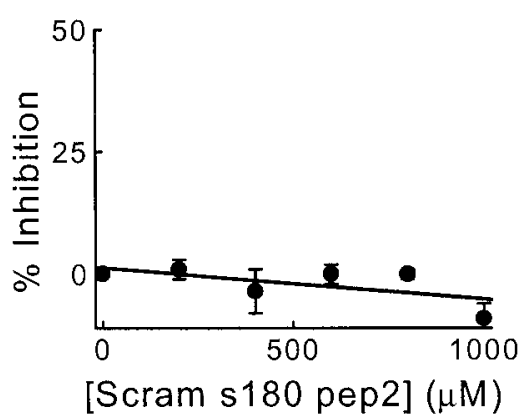

Figure 6. A peptide from the clathrin assembly domain of squid AP180 (s180 pep) inhibits clathrin assembly in vitro. $A$, The amount of inhibition of clathrin assembly depended on the concentration of s180 pep. Points represent the mean of three independent experiments, and error bars indicate SE. $B, C$, Two scrambled s180 peptides, Scram s180 pep1 and Scram s180 pep2, had no effect on clathrin assembly. Because none of the peptides inhibited by $>50 \%$ over this concentration range, these data were fit by a linear function (solid lines).

observed in synapses in which transmission was stable before injecting these proteins. The influence of the stability of basal synaptic transmission on the effects of injected proteins is shown in Figure 5. There was a strong correlation between the stability of basal synaptic transmission, measured as the rate of change in the baseline slope of the PSPs, and the effect of injected sC45 (Fig. $5 A$ ) or mC58 (Fig. 5B) on PSPs. In contrast, injections of GST (Fig. $5 C$ ) did not enhance synaptic transmission significantly, even when baseline transmission was steeply declining. The difference between the effects of sC45 and GST was statistically significant ( $p<0.01$; Student's $t$ test) as was the difference between mC58 and GST $(p<0.1)$. Although we do not know the reason for the decline in basal synaptic transmission, we suspect that it is attributable to a high rate of spontaneous transmitter release and subsequent depletion of the readily releasable pool of synaptic vesicles (Charlton et al., 1982). Thus, the clathrin assembly domains of squid and mouse AP180 may enhance transmitter release when the terminal is highly active.
A

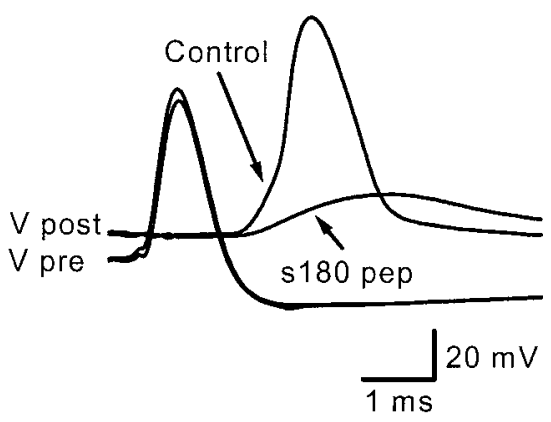

B

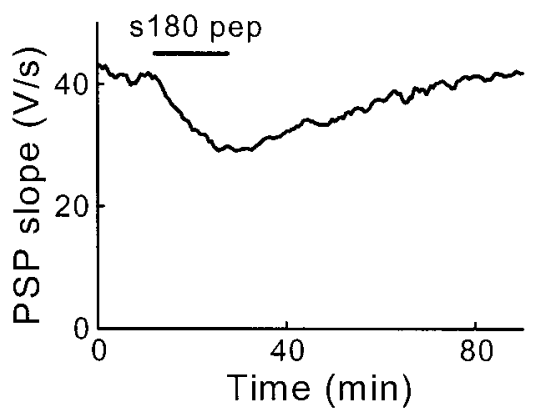

C

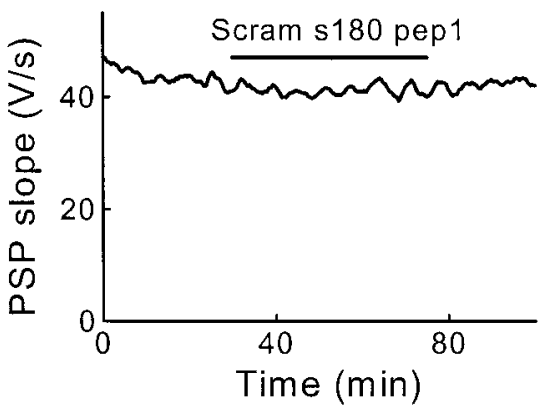

Figure 7. Sequence-specific inhibition of transmitter release by s180 pep. $A$, Superimposed traces of presynaptic and postsynaptic responses recorded before (Control) and during the injection of s180 pep. s180 pep reduced PSPs below the threshold for producing a postsynaptic action potential. $B$, The inhibitory effects of s180 pep were reversible after cessation of the peptide injection (during bar). C, Scram s180 pep1 had no effect on transmitter release.

\section{Clathrin assembly is essential for synaptic transmission}

As a second test of the in vivo role of clathrin assembly in synaptic transmission, we injected reagents that inhibit clathrin assembly by AP180. For this purpose we first considered the ability of a number of synthetic peptides from the well conserved blocks of the clathrin assembly domain of sAP180 to inhibit clathrin assembly in vitro. Among these peptides one (s180 pep) inhibited the assembly of bovine clathrin by sAP180. This inhibitory peptide was a 21-mer near the beginning of the sAP180 clathrin assembly domain, including amino acids 337-356. s180 pep produced a concentration-dependent block of clathrin assembly in vitro, with $38 \%$ inhibition observed at a peptide concentration of $1 \mathrm{~mm}$ (Fig. 6A). Two mutated versions of s180 pep, containing the same amino acids in a scrambled order (Scram s180 pep1 and Scram s180 pep2), had no effect on clathrin assembly in vitro at concentrations as high as $1 \mathrm{~mm}$ (Fig. $6 B, C$ ). These in vitro results suggest that s180 pep can be used in vivo to inhibit clathrin assembly by AP180, with the scrambled peptides serving as controls. 
Control

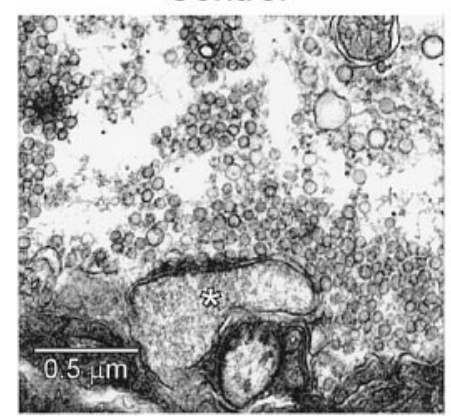

s180 pep

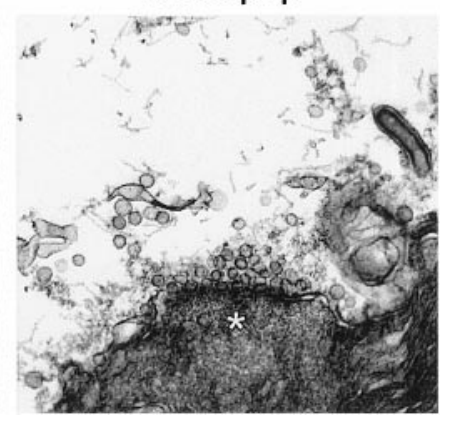

Figure 8. Presynaptic terminals injected with s180 pep are depleted of synaptic vesicles. Compared with terminals injected with inert control solutions $(A)$, s180 pep-injected terminals $(B)$ had drastically fewer synaptic vesicles. Asterisks mark postsynaptic spines. Scale bar in $A$ applies to both panels.

We next injected s180 pep into the squid giant presynaptic terminal to ask whether clathrin assembly by AP180 is necessary for synaptic vesicle endocytosis in vivo. If clathrin is important for endocytosis in the nerve terminal, then the block of clathrin assembly should have several effects: (1) prevent fused vesicular membrane from being retrieved from the plasma membrane and thereby increase presynaptic surface area, (2) inhibit the formation of clathrin-coated vesicles, (3) reduce the number of synaptic vesicles, and (4) inhibit transmitter release by reducing the number of synaptic vesicles. We found that s180 pep produced all of these lesions.

First, presynaptic injection of sAP180 pep inhibited evoked transmitter release without affecting the presynaptic action potential (Fig. 7A). On average, sAP180 pep injection inhibited PSPs by $36 \%(n=17$; Table 2$)$, although injection of a sufficient amount of this peptide (estimated to be several millimolar) completely inhibited synaptic transmission. Inhibition of the PSP was reversible over time (Fig. $7 B$ ), indicating that the inhibition was not attributable to damage of the presynaptic terminal. Scram s180 pep1, which had no effect on clathrin assembly in vitro, also had no effect on transmitter release (Fig. $7 C$, Table 2). This indicates that the inhibition of synaptic transmission seen with s180 pep is sequence-specific and that clathrin assembly in vivo by AP180 is essential for neurotransmitter release.

We next used electron microscopy to examine the ultrastructure of presynaptic terminals injected with s180 pep. During the injection of s180 pep, synaptic transmission was monitored and the terminals were fixed after the peptide had inhibited PSP slope by $80 \%$ or more. Control terminals were treated similarly, except that they were injected with a similar volume of inert solution that did not inhibit synaptic transmission. The two terminals injected with s180 pep were depleted of synaptic vesicles dramatically in comparison to the two control terminals (Fig. 8). This effect of s180 pep was quantified by measuring the spatial distribution of synaptic vesicles in the immediate vicinity of the active zone (Fig. $9 A$ ), using an approach described previously (Hess et al., 1993). For each 50-nm-wide shell within the presynaptic terminal, s180 pep injection reduced the mean number of synaptic vesicles (Fig. $9 A$ ). s180 pep caused a larger depletion of the synaptic vesicles that were farthest from the plasma membrane. This was analyzed by calculating the relative number of vesicles within each shell, determined by dividing the mean number of vesicles in each shell of the s180 pep-injected terminals by the mean number measured in the control terminals. This analysis showed that the vesicles within $100 \mathrm{~nm}$ of the plasma membrane were reduced by only $18 \%$, whereas those farther away were reduced by $82 \%$ (Fig. 9B). Integrated over the volume within $500 \mathrm{~nm}$ of the plasma membrane (Fig. 9C), the number of synaptic vesicles in s180 pepinjected terminals was reduced by $74 \%$, a statistically significant reduction ( $p<0.05$; Student's $t$ test). This reduction in the number of synaptic vesicles is a second indication that s180 pep inhibits endocytosis and suggests that s180 pep inhibits transmitter release by blocking clathrin assembly by AP180 in vivo.

The number of coated vesicles also was measured in presynaptic terminals injected with s180 pep or control terminals. In control terminals we observed approximately one coated vesicle per active zone, indicating that these structures are rather infrequent. However, terminals injected with s180 pep had virtually no coated vesicles, with their mean number of coated vesicles reduced by $84 \%$ as compared with the controls (Fig. 9D). This reduction in the number of coated vesicles in the presence of s180 pep is consistent with the block of clathrin assembly produced by s180 pep in vitro and is a third indication that clathrin assembly is necessary for synaptic vesicle endocytosis.

Finally, if s180 pep prevents endocytosis, then fused vesicular membrane should accumulate in the plasma membrane. We examined this by using electron microscopy to measure the perimeter of the nerve terminals injected with s180 pep or control solutions. Then these measurements were converted to membrane surface area by multiplying the perimeter by the thickness of the terminal section (Heuser and Reese, 1973; Burns et al., 1998). In control terminals the surface area of the presynaptic plasma membrane and synaptic vesicles was similar to values reported previously (Burns et al., 1998). However, terminals injected with s180 pep had a $20 \%$ larger plasma membrane surface area than the plasma membranes of the control terminals (Fig. $9 E ; p<0.05)$. This increased plasma membrane area suggests that the vesicular membrane that was lost after s180 pep injection may have been trapped in the plasma membrane. To test this hypothesis further, we measured the combined areas of synaptic vesicles, coated vesicles, and presynaptic plasma membrane for control terminals and those injected with s180 pep. The total area of membrane in these compartments did not change between s180 peptide-injected terminals and controls $(p=0.17)$, indicating that the expansion of the plasma membrane can, in large part, account for the loss in synaptic vesicle area after s180 pep injection. This is a fourth indication that s180 pep inhibited synaptic vesicle endocytosis and is consistent with a block of clathrin assembly by AP180 in vivo. We therefore conclude that preventing clathrin assembly by AP180 blocks synaptic vesicle endocytosis, which is predicted by the model that synaptic vesicle endocytosis requires clathrin.

It has been proposed that synaptic vesicle volume is determined by AP180 (Ye and Lafer, 1995a; Zhang et al., 1998). To test this proposal, we measured the diameter of synaptic vesicles in control terminals and those injected with s180 pep. Synaptic vesicle diameters were described by Gaussian functions in both control (Fig. $9 F$ ) and s180 pep-injected terminals (Fig. 9G). The halfwidth of the size distributions did not change, being $24 \mathrm{~nm}$ for control vesicles and $22 \mathrm{~nm}$ for vesicles in terminals injected with s180 pep. However, the mean diameter of synaptic vesicles was significantly larger ( $p<0.0001$; Student's $t$ test) in s180 pepinjected terminals $(70.0 \mathrm{~nm} ; n=909 \mathrm{SVs})$ than in control terminals $(66.7 \mathrm{~nm} ; n=939 \mathrm{SVs})$. Thus, s180 pep caused a shift in the vesicle size distribution without changing the shape of the distri- 
Figure 9. s180 pep inhibits endocytosis A in the squid giant presynaptic terminal. $A$, Spatial distribution of synaptic vesicles in terminals injected with s180 pep or control solutions. Error bars indicate means and SE values for 376 active zones from two terminals injected with s180 pep and 209 active zones from three control terminals. $B$, Relative spatial distribution of synaptic vesicles, determined from the data in $A$ by dividing the means for s 180 pep by those for control terminals. The dashed line indicates a ratio of 1 , representing no effect of s180 pep. $C, D$, Mean number of synaptic vesicles $(C)$ and coated vesicles $(D)$ in terminals injected with $\mathrm{s} 180$ pep or control solution. $E$, Mean areas of membrane in synaptic vesicles $(S V)$, coated vesicles $(C V)$, and plasma membrane $(P M)$ in sections taken from terminals injected with s180 pep or control solution. $F-H$, Distribution of synaptic vesicle $(S V)$ diameters in terminals injected with control solution $(F)$ or s180 pep $(G)$. The curved lines indicate Gaussian functions fit to these distributions. $H$ Superimposition of the two distributions, with s180 pep measurements in black.

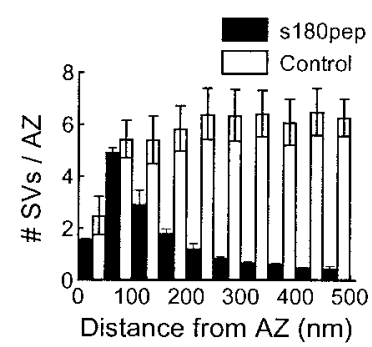

$\mathbf{B}$

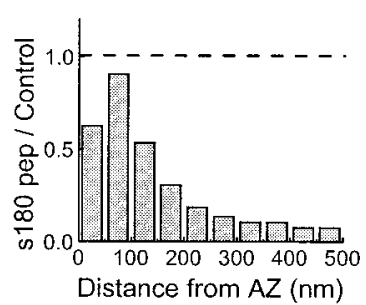

C

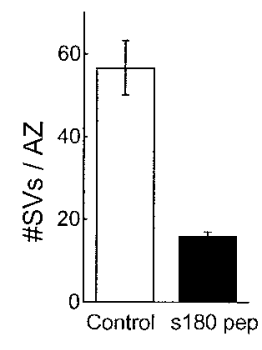

$\mathrm{E}$

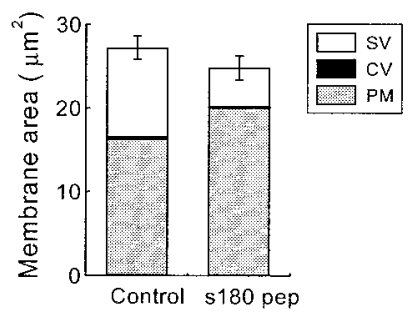

$\mathbf{F}$

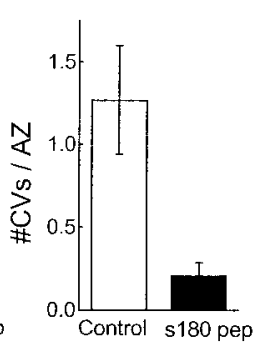

$\mathbf{G}$
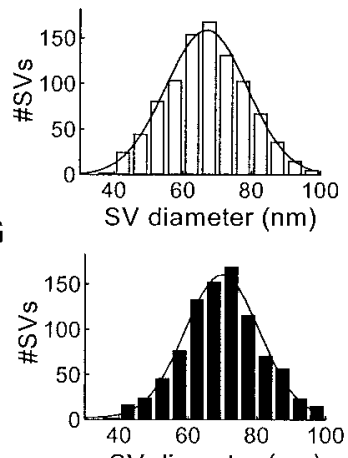

H

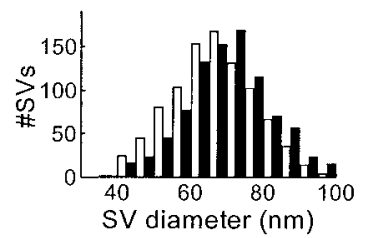

bution (Fig. 9H). The change in vesicle diameter corresponds to a $16 \%$ increase in volume, indicating that clathrin assembly by AP180 influences the volume of synaptic vesicles.

\section{DISCUSSION}

We have used the squid giant synapse to study the function of AP180, a synapse-specific protein that assembles clathrin in vitro. The squid homolog of AP180 exhibits strong biochemical similarities to mammalian AP180 despite low conservation of the C-terminal clathrin assembly domain of these proteins. Presynaptic injection of the clathrin assembly domain of AP180 enhanced transmitter release from terminals in which transmission was declining. Conversely, preventing clathrin assembly by injecting a peptide from the clathrin assembly domain of AP180 inhibited transmitter release and prevented synaptic vesicle endocytosis. These results indicate that clathrin, and its assembly by AP180, is necessary for synaptic vesicle trafficking.

\section{Functional conservation of the clathrin assembly domain of AP180}

Cloning of a new AP180 family member allowed us to perform a detailed phylogenetic analysis that revealed several new insights into this family. First, it appears that the ubiquitously expressed vertebrate AP180-2 subfamily arose at an earlier point in evolution than the neuronal-specific vertebrate AP180-1 subfamily (see Fig. 2). Second, it appears that whereas all of the AP180 family members are well conserved in their $\sim 33 \mathrm{kDa}$ N-terminal domains, they have diverged considerably in their variably sized C-terminal domains (see Fig. 1, Table 1). Although the C-terminal domain of mouse AP180 is known to assemble clathrin into homogeneously sized cages in vitro (Ye and Lafer, 1995a,b), it was not clear whether the variable C-terminal domains of other AP180 family members would assemble clathrin. We found that both full-length squid AP180 and the C-terminal domain of squid AP180 could assemble clathrin in vitro into a homogeneously sized population of cages despite their divergent C-terminal domains (see Fig. 3). This is consistent with a report that the C-terminal domain of $S$. cerevisiae AP180a can bind to clathrin, although it was not known whether this protein could assemble clathrin (Wendland and Emr, 1998). Our studies pro- vide strong support for the functional conservation of the evolutionarily divergent C-terminal domain of AP180 family members and predict that all members of the family can assemble clathrin.

The domains responsible for the various ligand-binding properties of mammalian AP180 have been well defined biochemically (Ye and Lafer, 1995b; Ye et al., 1995). The N-terminal domain binds to inositides (Ye et al., 1995), and this binding event inhibits clathrin assembly (Norris et al., 1995; Ye et al., 1995; Hao et al., 1997). The C-terminal domain is responsible for the clathrin assembly activity (Ye and Lafer, 1995b). Despite a detailed description of the clathrin assembly properties of mammalian AP180 in vitro, nothing was known about the functions of these domains in vivo. For this reason, we injected various AP180 protein domains into the squid giant presynaptic terminal. Although injection of the $\mathrm{C}$-terminal clathrin assembly domains of either mouse AP180 (mC58) or squid AP180 (sC45) enhanced synaptic transmission (see Fig. 4, Table 2), the N-terminal domain $(\mathrm{mN} 33)$ and central domain (mM42) had no effect. This correlation between clathrin assembly properties and their effects on synaptic transmission suggests that these domains enhance clathrin assembly in vivo, as predicted by their in vitro properties. It is possible that the enhancement of synaptic transmission by $\mathrm{mC} 58$ is attributable to AP180 binding other endocytotic proteins, such as $\mathrm{EH}$ domain proteins or AP2. However, mC58 does not contain the NPF motifs thought to be important for proteins binding to EH domains (Salcini et al., 1997), suggesting that interactions with $\mathrm{EH}$ domains are not responsible for the enhancement of synaptic transmission. It is also unlikely that the effect is attributable to an interaction between AP180 and AP2, because neither mM42 nor mAP180 had an effect on transmission (see Table 2), yet they both contain the AP-2 binding site (Hao et al., 1999). Therefore, it is likely that $\mathrm{mC} 58$ and $\mathrm{sC} 45$ enhance synaptic transmission by binding to clathrin and stimulating clathrindependent endocytosis. Microinjection of full-length squid or full-length mouse AP180 had no effect on synaptic transmission (see Table 2), although these constructs included the C-terminal clathrin assembly domains. It is possible that this was attributable to the presence of the regulatory $\mathrm{N}$-terminal domain, which inhibits clathrin assembly (Ye et al., 1995; Hao et al., 1997). 


\section{Manipulating clathrin assembly by AP180 alters synaptic vesicle trafficking}

Our results provide several lines of support for a role for clathrin in synaptic vesicle trafficking. Our observation that clathrin assembly domains enhanced synaptic transmission is the first demonstration that stimulation of clathrin-mediated endocytosis can affect synaptic transmission. We presume that the enhancement arises from increasing the number of synaptic vesicles in the readily releasable pool of vesicles, attributable to the stimulation of vesicle endocytosis and subsequent reformation of synaptic vesicles. Consistent with this explanation, we found that the enhancement of synaptic transmission by these domains was largest in terminals in which basal synaptic transmission was declining. We interpreted this to suggest that the concentration of AP180 is only limiting in very active terminals, although alternative explanations cannot be excluded without understanding why basal synaptic transmission declines.

Stronger support for the hypothesis that clathrin is important for synaptic vesicle endocytosis comes from s180 pep, which prevents clathrin assembly in vitro (see Fig. 6). Injecting s180 pep into the squid presynaptic terminal inhibited synaptic transmission reversibly (see Fig. 7). The fact that synaptic transmission could be inhibited completely by s180 pep indicates that clathrin assembly by AP180 is essential for synaptic transmission. Further, s180 pep injection reduced the number of synaptic vesicles and coated vesicles and increased the surface area of the presynaptic terminal (see Fig. 9). All of these changes indicate that impairment of clathrin assembly prevents synaptic vesicle endocytosis. Our results complement recent work showing that mutation of the Drosophila AP180 gene impairs synaptic transmission and depletes synaptic vesicles (Zhang et al., 1998). Because Drosophila AP180 is not known to assemble clathrin, it was not clear whether the resulting phenotype represents the loss of clathrin assembly activity or some other function of AP180. Our results suggest that lack of clathrin assembly is the likely explanation for the consequences of genetic deletion of AP180.

Based on the common definition that docked vesicles are those closest to the plasma membrane (Schweizer et al., 1995), s180 pep caused the number of docked synaptic vesicles to decrease by $\sim 40 \%$ (see first column, Fig. 9B), yet evoked transmitter release completely stopped. There are several possible explanations for the inability of the remaining docked vesicles to fuse. It is possible that these vesicles are generated by a pathway incapable of correctly sorting vesicular proteins, so these vesicles do not contain proteins required for fusion. Alternatively, transmitter release could be disrupted by accumulation of vesicular membrane in the plasma membrane. Regardless of the specific mechanism, our results indicate that nerve terminals cannot sustain transmitter release in the absence of clathrin-mediated endocytosis.

\section{Molecular pathways for synaptic vesicle recycling}

A role for clathrin-dependent endocytosis in synaptic vesicle trafficking originally was suggested by Heuser and Reese (1973). Because their experiments used heavy and possibly nonphysiological rates of synaptic activity, it has been suggested that clathrin-independent mechanisms may be involved in vesicle trafficking under more physiological conditions (for review, see Palfrey and Artalejo, 1998). Our experiments argue strongly to the contrary by showing that clathrin-mediated endocytosis is important even during physiological levels of synaptic activity. However, terminals injected with s180 pep still had approximately one-fourth of their synaptic vesicles remaining. It is possible that the peptide may not have inhibited clathrin-mediated endocytosis completely, allowing other clathrin assembly proteins, such as AP2, to sustain endocytosis at a low level. This also could account for the presence of a few coated vesicles in peptide-injected terminals (see Fig. 9D) and the existence of synaptic vesicles in Drosophila AP180 mutants (Zhang et al., 1998). Alternatively, the remaining synaptic vesicles may have been endocytosed via a second, clathrin-independent pathway (Koenig and Ikeda, 1996; Klingauf et al., 1998; Kuromi and Kidokoro, 1998; Shi et al., 1998).

Clathrin cages assembled in vitro in the presence of mammalian AP180 are smaller and more uniform than those assembled without AP180, suggesting that AP180 may control the volume of synaptic vesicles (Ye and Lafer, 1995a). Support for this hypothesis comes from our finding that the synaptic vesicles remaining in terminals injected with s180 pep had a $16 \%$ larger mean volume than control terminals (see Fig. $9 F-H$ ), as well as observations that mutation of the AP180 gene reduces vesicle volume (Zhang et al., 1998; Nonet et al., 1999). These results indicate that synaptic vesicles of normal size require AP180 and, hence, clathrin-coated vesicles. However, the source of these vesicles is still uncertain. Coated vesicles clearly bud off from the plasma membrane (Heuser and Reese, 1973; Takei et al., 1996), and interfering with AP180 must prevent such budding because s180 pep increases presynaptic plasma membrane area. However, it is also possible that coated vesicles bud off from endosomes (Heuser and Reese, 1973; Schweizer et al., 1995; Sudhof, 1995). Thus, our results cannot resolve the long-standing question of whether clathrin-based budding from endosomes is responsible for the generation of synaptic vesicles (Takei et al., 1996; Murthy and Stevens, 1998; Shi et al., 1998).

In summary, our results show that clathrin assembly by AP180 is important for endocytosis in nerve terminals experiencing physiological amounts of activity. AP180 also is essential for neurotransmitter release and for the formation of synaptic vesicles of normal size. Our results support a clathrin-based mechanism for synaptic vesicle recycling, although it remains to be determined whether an endosomal intermediate is involved in endocytosis and why some synaptic vesicles are present when AP180-mediated clathrin assembly is impaired. Although clathrin-based endocytosis is the predominant mechanism of endocytosis, it is still possible that clathrin-independent mechanisms participate in synaptic vesicle endocytosis.

\section{REFERENCES}

Ahle S, Ungewickell E (1986) Purification and properties of a new clathrin assembly protein. EMBO J 5:3143-3149.

Augustine GJ, Burns ME, DeBello WM, Pettit DL, Schweizer FE (1996) Exocytosis: proteins and perturbations. Annu Rev Pharmacol Toxicol 36:659-701.

Bommert K, Charlton MP, DeBello WM, Chin GJ, Betz H, Augustine GJ (1993) Inhibition of neurotransmitter release by C2-domain peptides implicates synaptotagmin in exocytosis. Nature 363:163-165.

Burns ME, Augustine GJ (1999) Functional studies of presynaptic proteins at the squid giant synapse. In: Neurotransmitter release: frontiers in molecular biology (Bellen H, ed), 237-264. New York: Oxford UP.

Burns ME, Sasaki T, Takai Y, Augustine GJ (1998) Rabphilin-3A: a multifunctional regulator of synaptic vesicle traffic. J Gen Physiol 111:243-255.

Ceccarelli B, Hurlbut WP, Mauro A (1973) Turnover of transmitter and synaptic vesicles at the frog neuromuscular junction. J Cell Biol 57:499-524.

Ceccarelli B, Grohovaz F, Hurlbut WP (1979) Freeze-fracture studies of frog neuromuscular junctions during intense release of neurotransmitter. II. Effects of electrical stimulation and high potassium. J Cell Biol 81:178-192. 
Charlton MP, Smith SJ, Zucker RS (1982) Role of presynaptic calcium ions and channels in synaptic facilitation and depression at the squid giant synapse. J Physiol (Lond) 323:173-193.

Cremona O, De Camilli P (1997) Synaptic vesicle endocytosis. Curr Opin Neurobiol 7:323-330.

De Camilli P, Takei K (1996) Molecular mechanisms in synaptic vesicle endocytosis and recycling. Neuron 16:481-486.

Dreyling MH, Martinez-Climent JA, Zheng M, Mao J, Rowley JD, Bohlander SK (1996) The $t(10 ; 11)(p 13 ; q 14)$ in the U937 cell line results in the fusion of the AF10 gene and CALM, encoding a new member of the AP-3 clathrin assembly protein family. Proc Natl Acad Sci USA 93:4804-4809.

Felsenstein J (1985) Confidence limits on phylogenies: an approach using the bootstrap. Evolution 39:783-791.

Hao W, Tan Z, Prasad K, Reddy KK, Chen J, Prestwich GD, Falck JR, Shears SB, Lafer EM (1997) Regulation of AP-3 function by inositides. Identification of phosphatidylinositol 3:4,5-trisphosphate as a potent ligand. J Biol Chem 272:6393-6398.

Hao W, Luo Z, Zheng L, Prasad K, Lafer EM (1999) AP180 and AP2 interact directly in a complex that cooperatively assembles clathrin. J Biol Chem 274:22785-22794.

Hess SD, Doroshenko PA, Augustine GJ (1993) A functional role for GTP-binding proteins in synaptic vesicle cycling. Science 259:1169-1172.

Heuser JE, Reese TS (1973) Evidence for recycling of synaptic vesicle membrane during transmitter release at the frog neuromuscular junction. J Cell Biol 57:315-344.

Ishikawa K, Nagase T, Suyama M, Miyajima N, Tanaka A, Kotani H, Nomura N, Ohara O (1998) Prediction of the coding sequences of unidentified human genes. X. The complete sequences of 100 new cDNA clones from brain which can code for large proteins in vitro. DNA Res 5:169-176.

Keen JH (1987) Clathrin assembly proteins: affinity purification and a model for coat assembly. J Cell Biol 105:1989-1998.

Klingauf J, Kavalali ET, Tsien RW (1998) Kinetics and regulation of fast endocytosis at hippocampal synapses. Nature 394:581-585.

Koenig JH, Ikeda K (1996) Synaptic vesicles have two distinct recycling pathways. J Cell Biol 135:797-808.

Kuromi H, Kidokoro Y (1998) Two distinct pools of synaptic vesicles in single presynaptic boutons in a temperature-sensitive Drosophila mutant, shibire. Neuron 20:917-925.

Lindner R, Ungewickell E (1992) Clathrin-associated proteins of bovine brain coated vesicles. An analysis of their number and assemblypromoting activity. J Biol Chem 267:16567-16573.

Maycox PR, Link E, Reetz A, Morris SA, Jahn R (1992) Clathrincoated vesicles in nervous tissue are involved primarily in synaptic vesicle recycling. J Cell Biol 118:1379-1388.

McMahon HT (1999) Endocytosis: an assembly protein for clathrin cages. Curr Biol 9:R332-R335.

Morris SA, Schroder S, Plessmann U, Weber K, Ungewickell E (1993) Clathrin assembly protein AP180: primary structure, domain organization, and identification of a clathrin binding site. EMBO J 12:667-675.

Murthy VN, Stevens CF (1998) Synaptic vesicles retain their identity through the endocytic cycle. Nature 392:497-501.

Nonet ML, Holgado AM, Brewer F, Serpe CJ, Norbeck BA, Holleran J, Wei L, Hartwieg E, Jorgensen EM, Alfonso A (1999) UNC-11, a Caenorhabditis elegans AP180 homologue, regulates the size and protein composition of synaptic vesicles. Mol Biol Cell 10:2343-2360.

Norris FA, Ungewickell E, Majerus PW (1995) Inositol hexakisphosphate binds to clathrin assembly protein 3 (AP-3/AP180) and inhibits clathrin cage assembly in vitro. J Biol Chem 270:214-217.

Palfrey HC, Artalejo CR (1998) Vesicle recycling revisited: rapid endocytosis may be the first step. Neuroscience 83:969-989.

Pearse BM (1976) Clathrin: a unique protein associated with intracellular transfer of membrane by coated vesicles. Proc Natl Acad Sci USA 73:1255-1259.
Perry DG, Hanson V, Benuck ML, Puszkin S (1991) Neuronal protein NP185 in avian and murine cerebellum: expression during development and evidence for its presence in nerve endings. J Histochem Cytochem 39:1461-1470.

Perry DG, Li S, Hanson V, Puszkin S (1992) Neuromuscular junctions contain NP185: the multifunctional protein is located at the presynaptic site. J Neurosci Res 33:408-417.

Prasad K, Lippoldt RE (1988) Molecular characterization of the AP180 coated vesicle assembly protein. Biochemistry 27:6098-6104.

Rothman JE (1994) Mechanisms of intracellular protein transport. Nature 372:55-63.

Salcini AE, Confalonieri S, Doria M, Santolini E, Tassi E, Minenkova O, Cesareni, G, Pelicci PG, Di Fiore PP (1997) Binding specificity and in vivo targets of the $\mathrm{EH}$ domain, a novel protein-protein interaction module. Genes Dev 11:2239-2249.

Sanchez ME, Nuno CM, Buchanan J, Augustine GJ (1990) Contractions of the squid stellate ganglion. J Exp Biol 152:369-387.

Scheller RH (1995) Membrane trafficking in the presynaptic nerve terminal. Neuron 14:893-897.

Schweizer FE, Betz H, Augustine GJ (1995) From vesicle docking to endocytosis: intermediate reactions of exocytosis. Neuron 14:689-696.

Shi G, Faundez V, Roos J, Dell'Angelica EC, Kelly RB (1998) Neuroendocrine synaptic vesicles are formed in vitro by both clathrin-dependent and clathrin-independent pathways. J Cell Biol 143:947-955.

Sousa R, Tannery NH, Zhou S, Lafer EM (1992) Characterization of a novel synapse-specific protein. I. Developmental expression and cellular localization of the F1-20 protein and mRNA. J Neurosci 12:2130-2143

Sudhof TC (1995) The synaptic vesicle cycle: a cascade of proteinprotein interactions. Nature 375:645-653.

Swofford DL (1998) PAUP*. Phylogenetic analysis using parsimony (*and other methods), Version 4. Sunderland, MA: Sinauer.

Takei K, Mundigl O, Daniell L, De Camilli P (1996) The synaptic vesicle cycle: a single vesicle budding step involving clathrin and dynamin. J Cell Biol 133:1237-1250.

Thompson JD, Higgins DG, Gibson TJ (1994) CLUSTAL W: improving the sensitivity of progressive multiple sequence alignment through sequence weighting, position-specific gap penalties, and weight matrix choice. Nucleic Acids Res 22:4673-4680.

Wendland B, Emr SD (1998) Pan1p, yeast eps15, functions as a multivalent adaptor that coordinates protein-protein interactions essential for endocytosis. J Cell Biol 141:71-84.

Ye W, Lafer EM (1995a) Bacterially expressed F1-20/AP-3 assembles clathrin into cages with a narrow size distribution: implications for the regulation of quantal size during neurotransmission. J Neurosci Res 41:15-26.

Ye W, Lafer EM (1995b) Clathrin binding and assembly activities of expressed domains of the synapse-specific clathrin assembly protein AP-3. J Biol Chem 270:10933-10939.

Ye W, Ali N, Bembenek ME, Shears SB, Lafer EM (1995) Inhibition of clathrin assembly by high affinity binding of specific inositol polyphosphates to the synapse-specific clathrin assembly protein AP-3. J Biol Chem 270:1564-1568

Young JZ (1939) Fused neurons and synaptic contacts in the giant nerve fibres in cephalopods. Philos Trans R Soc Lond [Biol] 229:465-503.

Zhang B, Koh YH, Beckstand RB, Budnik V, Ganetzky B, Bellen HJ (1998) Synaptic vesicle size and number are regulated by a clathrin adaptor protein required for endocytosis. Neuron 21:1465-1475.

Zhou S (1994) Molecular characterization of F1-20: a novel synapsespecific clathrin assembly protein. $\mathrm{PhD}$ dissertation, University of Pittsburgh.

Zhou S, Sousa R, Tannery NH, Lafer EM (1992) Characterization of a novel synapse-specific protein. II. cDNA cloning and sequence analysis of the F1-20 protein. J Neurosci 12:2144-2155.

Zhou S, Tannery NH, Yang J, Puszkin S, Lafer EM (1993) The synapsespecific phosphoprotein F1-20 is identical to the clathrin assembly protein AP-3. J Biol Chem 268:12655-12662. 